\title{
BMJ Open Clinical efficacy, safety and tolerability of aliskiren monotherapy: a protocol for an umbrella review
}

\author{
Qiyuan Zhao (D , , ${ }^{1,2}$ Jiantong Shen, ${ }^{2}$ Jingya Lu, ${ }^{2}$ Fan Li, ${ }^{3}$ Qi Jiang, ${ }^{2}$ Yuanyuan Wang ${ }^{1}$
}

To cite: Zhao Q, Shen J, Lu J, et al. Clinical efficacy, safety and tolerability of aliskiren monotherapy: a protocol for an umbrella review. BMJ Open 2020;10:e033448. doi:10.1136/ bmjopen-2019-033448

- Prepublication history for this paper is available online. To view these files, please visit the journal online (http://dx.doi org/10.1136/bmjopen-2019033448).

Received 07 August 2019 Revised 29 December 2019 Accepted 31 December 2019

Check for updates

(c) Author(s) (or their employer(s)) 2020. Re-use permitted under CC BY-NC. No commercial re-use. See rights and permissions. Published by BMJ.

${ }^{1}$ School of Nursing, Huzhou University, Huzhou Central Hospital, Huzhou, China ${ }^{2}$ School of Medicine, Huzhou University, Huzhou Central Hospital, Huzhou, China

${ }^{3}$ College of Nursing, University of Missouri System, St. Louis, Missouri, USA

Correspondence to Jiantong Shen; sjiantong@163.com

\section{ABSTRACT}

Introduction Aliskiren is a newly developed medicine. As one of the effective renin-angiotensin-aldosterone system inhibitors, its role in lowering blood pressure has been recognised. However, its safety and tolerability still remain controversial. The aim of the paper is to systematically summarise the published studies about the clinical efficacy and side effects of aliskiren monotherapy.

Methods and analysis A comprehensive review of PubMed, Embase and Cochrane Library databases published from inception until June 2019 will be conducted. The selected articles are meta-analyses that integrated the randomised controlled studies, which evaluated efficacy, safety and tolerability of aliskiren monotherapy. Two people will select eligible articles and extract data independently. Any disputes will be resolved by discussion or the arbitration of a third person. The quality of reporting evidence will be assessed using the AMSTAR 2 tool. Study selection process will be presented using a flowchart. We will re-analyse each outcome with the random effect methods if necessary. If possible, we will also calculate $95 \%$ prediction intervals for each random effect estimate, by using Egger's test to evaluate if the reporting bias existed.

Ethics and dissemination Ethical approval is not required for the study, as we only collected data from available published materials. This umbrella review will be also submitted to a peer-reviewed journal for publication after completion.

PROSPERO registration number CRD42019142141.

\section{INTRODUCTION}

\section{Description of the condition}

Hypertension is a highly prevalent disease and its treatment and control are less than satisfactory. The prevalence of hypertension in the USA (defined as taking antihypertensive medication or having a systolic pressure of $\geq 140 \mathrm{~mm} \mathrm{Hg}$ and/or a diastolic pressure of $\geq 90 \mathrm{~mm} \mathrm{Hg}$ ) was approximately $32 \%$ and had remained relatively constant since $1999 .{ }^{1-3}$ Given its high prevalence, hypertension will increase the risk for heart disease and stroke, which are two of the leading causes of death of Americans. ${ }^{4}$

Heart failure (HF) is a rapidly growing public health problem. The estimated

\section{Strengths and limitations of this study}

- This will be the first study that systematically summarises the effectiveness, safety and tolerability of aliskiren monotherapy clinically.

- When sufficient data are available, we will stratify our comparisons by dosage or specific categories of the medications.

- If more than one meta-analysis evaluates the same outcome when comparing aliskiren with the same drug, we will incorporate all the original trials from those meta-analyses, without including duplicates.

- If the included reviews in our study are not of high quality, we will re-analyse each outcome using the random effects model.

- The methodological quality of the eligible reviews will be evaluated using AMSTAR 2 for assessing risk of bias.

prevalence of it is $>37.7$ million individuals globally, imposing a great burden to society. ${ }^{5}$ $\mathrm{HF}$ is also a leading cause of hospitalisation among adults and the elderly. In the USA, medical expenses for patients with $\mathrm{HF}$ are expected to rise from US $\$ 20.9$ billion in 2012 to US $\$ 53.1$ billion by $2030 .{ }^{6} \mathrm{HF}$ is usually associated with diabetes and patients, with both conditions having a progressive chronic disease trajectory. ${ }^{7}$ Renin-angiotensin-aldosterone system (RAAS) inhibitors are used for treatment of cardiovascular and renal diseases.

\section{Description of the intervention}

RAAS blockade is an effective approach to interfere with the pathogenesis of hypertension, cardiovascular disease and chronic renal failure. ${ }^{8}$ RAAS inhibitors fall into five distinctive drug classes: beta blockers, renin inhibitors, angiotensin receptor enzyme inhibitors (ACEIs), angiotensin receptors (ARBs) and aldosterone inhibitors. ${ }^{10}$

Aliskiren is the first in a new class of oral, non-peptide, low molecular weight direct renin inhibitor. Because of its mechanism of decreasing Ang I, aliskiren can lower blood 


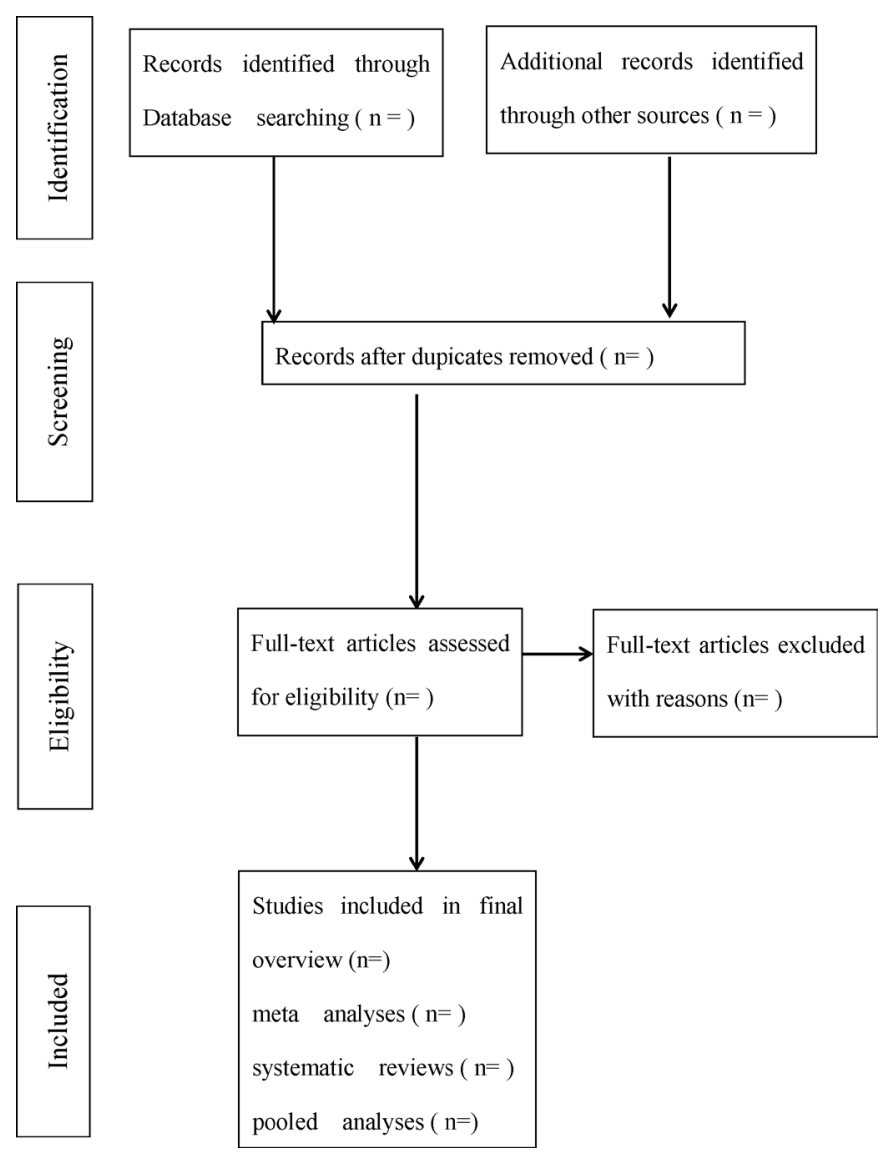

Figure 1 Flow diagram of study selection process.

pressure (BP).$^{11}$ It had already been approved by the US Food and Drug Administration for the management of hypertension by $2007 .{ }^{10}$ Furthermore, aliskiren has been proven to be beneficial in the management of congestive $\mathrm{HF}$ and proteinuria either as monotherapy, ${ }^{12}{ }^{13}$ or in combination with ACEIs or ARBs. ${ }^{14} 15$

Several systematic reviews, ${ }^{16}{ }^{17}$ meta-analyses ${ }^{18-20}$ and pooled analyses ${ }^{21}$ have evaluated the clinical outcomes of aliskiren treatment. Some articles reported favourable clinical outcomes, ${ }^{18}{ }^{21}$ whereas many others ${ }^{16} 171920$ reported that aliskiren had no significant outcomes. For instance, in Bangalore's review, ${ }^{16}$ when compared with placebo, the ARBs or aldosterone antagonist, aliskiren did not result in significant changes in the rate of efficacy outcomes (the outcome of death, cardiovascular death, composite outcome of cardiovascular death or heart failure hospitalisation). Furthermore, when compared with placebo, aliskiren increased the risk of hypotension. In Zhang s ${ }^{19}$ review, aliskiren monotherapy had no effect on major cardiovascular events, death, cardiac death, myocardial infarction or stroke. An umbrella review evaluating the clinical effectiveness, safety and tolerability of aliskiren is required to weigh the pros and cons of aliskiren monotherapy in the literature.

\section{Aim}

The aim of the study is to systematically overview the published articles about the clinical efficacy and side effects of aliskiren monotherapy.

\section{METHODS}

\section{Stage 1: identifying relevant studies}

At this stage, authors will establish a team and discuss the eligibility criteria, electronic databases and search strategy.

\section{Eligibility criteria}

The selected articles will meet the following requirements: published in English; meta-analysis, systematic review or pooled analysis; meta-analyses that integrated the randomised controlled studies, which evaluated efficacy, tolerability or safety of aliskiren treatment and metaanalyses that had substantial data and were upto-date.

The articles meeting the following requirements will be excluded: observational studies, conference briefs, editorials or overview articles; meta-analyses in which the intervening measures only included aliskiren combination therapy. Meta-analyses that did not provide specific study data (number of incident events, number of study population, follow-up period, relative risks and $95 \% \mathrm{CIs}$ ) and in which the missing data was not retrievable from the original studies will be excluded.

\section{Databases}

The identification of studies relevant to this overview will be gained by searching of the published reviews from inception of databases until June 2019 which are listed below: PubMed, Embase and Cochrane Library. We will also manually search all reference lists of the included studies to identify additional reviews of relevance.

\section{Search strategy}

We will use the search strategy with those specified keywords: (aliskiren OR direct renin inhibitor OR reninangiotensin inhibition OR spp100 OR takturna) AND (systematic review OR meta-analysis OR pooled analysis). We will modify the search strategy to suit all three databases.

\section{Stage 2: study selection}

We will import our search results into Endnote software and then start selection. The review process consists of two levels of screening: (1) a title and abstract review and (2) a full-text review. In the first phase of screening, the titles and abstracts of the retrieved articles will be browsed through and analysed by two independent investigators to identify potential eligibility. In the second phase, the two investigators will then independently evaluate the full-text articles to decide whether each should be included/excluded. Any discrepancies in the two phases will be reconsidered, and unresolved disagreements will be further discussed with a third investigator, until a full 
consensus is reached. The outline of the study selection procedure will be shown in a flowchart (figure 1).

\section{Stage 3: data extraction}

Data extraction tables will be established in Microsoft software and the data from selected articles will be extracted independently by two people. Disputes will be resolved by discussion or the arbitration of a third person if necessary. The following categories of data will be extracted: the first author, year of publication, number of trials included, main population, comparative drugs, follow-up period and the investigated clinical outcomes.

\section{Population}

Hypertension or patients with HF, some of them suffered from diabetes and/or nephropathy or albuminuria at the same time.

\section{Intervention}

Including aliskiren monotherapy.

\section{Comparators}

Conventional anti-hypertension and HF medications, such as ARBs, ACEIs, hydrochlorothiazide.

\section{Type of studies}

Systematic reviews, meta analyses or pooled analyses that integrated randomised controlled trials

\section{Outcomes}

Primary outcomes

Cardiovascular outcomes such as mortality rate, the composite of death and major adverse events, the incidence of stroke and myocardial infarction.

\section{Secondary outcomes}

Changes of surrogate markers such as NT-proBNP, BNP levels, plasma renin activity, plasma renin concentration.

Renal outcomes such as the incidence of hyperkalemia, changes of serum creatinine or estimated glomerular filtration rate, changes of urinary albumin creatinine ratio, urinary albumin excretion ratio, urinary protein creatinine rate and urinary protein excretion rate.

Antihypertensive effects such as $\mathrm{BP}$ reduction, BP response rate, $\mathrm{BP}$ control rate, the incidence of some adverse events.

Cost-effectiveness results such as incremental costeffectiveness ratios, average cost-effectiveness ratio, benefit-cost ratio and unit costs.

The study of specific risk estimations will also be included: risk ratio, OR, weighted mean difference, standard mean difference together with their 95\% CI and number of incident events and total events in each study. If more than one meta-analysis evaluates the same outcome when comparing aliskiren with the same drug, we will incorporate all the original trials from those metaanalyses, without including duplicates. We will summarise all the available data to get a more comprehensive and objective result.

\section{Stage 4: data synthesis and statistical analysis}

Statistical analysis will be conducted with RevMan V.5.3 software provided by Cochrane Collaboration and Stata V.14.0 software. In our analysis, when possible, we will stratify the comparisons into several groups according to the dose, such as initial group, initial to high group, high group. In each group, the doses of aliskiren and control drug are comparable. We will also stratify the comparisons according to the categories of control drugs. For example, we will divide ARBs into three specific drugs: losartan, valsartan and irbesartan.

Given the totally different characteristics of our targeted population, we would also do subgroup analysis. For example, when evaluating cardiovascular effects, we will divide heart failure patients into three groups: normotensive, hypertensive and mixed population. We can also divide patients into: patients with no diabetes, patients with diabetes, patients with diabetes and high cardiovascular risk or established cardiovascular disease. When evaluating renal outcomes, we will divide patients into three groups: simple hypertension patients, patients with hypertension and diabetes mellitus type 1 or type 2; and patients who are suffering from hypertension, diabetes and nephropathy or albuminuria at the same time. When evaluating antihypertensive effects, we will divide patients into three groups: young patients $(<50$ years), early elderly patients (50-70 years), elderly patients ( $>70$ years).

For each outcome, if the random model was already used, we will record the results. If not, we will extract original data and reanalyse them with the random effect methods to get a new $95 \%$ CI, to increase credibility. We will also calculate $95 \%$ PI for each random effect estimate, to represent the range in which the effect estimates of future studies will lie. The $Q$ and $\mathrm{I}^{2}$ test statistics will be calculated to determine the amount of heterogeneity. For the $\mathrm{Q}$ statistic, $\mathrm{p}<0.05$ will be considered to indicate significant differences. An $\mathrm{I}^{2}$ statistic, $\mathrm{I}^{2}>50 \%$, indicates substantial heterogeneity, and $\mathrm{I}^{2}>75 \%$ indicates considerable heterogeneity.

If sufficient studies are included and they covered relevant information, we will also do the cost-effectiveness analysis, compared between the intervention and the control group. We will extract common outcome results such as incremental cost-effectiveness ratios, average costeffectiveness ratio, benefit-cost ratio and unit costs from the included studies. We will report outcome measures as presented in the original studies and, for comparison, we will convert the original values to international dollars using purchasing power parity for the country where the study is conducted.

\section{Stage 5: identifying possible publication bias}

If an outcome is examined in at least three articles, we will use Egger's test (conducted using Stata V.14.0) to evaluate if the reporting bias exists. Values of $\mathrm{p}<0.1^{22}$ will be interpreted as statistically significant. 


\section{Stage 6: evaluating quality of included studies}

The methodological quality of the eligible studies will be evaluated using the AMSTAR 2 tool for assessing risk of bias. AMSTAR 2 is a major revision of the original AMSTAR instrument, which was designed to appraise systematic reviews that included randomised controlled trials. AMTAR 2, like its predecessor, has an important role as a convenient teaching aid and a brief checklist for those conducting systematic reviews. AMSTAR 2, unlike its predecessor, has the capacity to identify critical weaknesses that reduce confidence in the findings of a review. ${ }^{23}$

\section{Patient and public involvement}

No patients are involved in developing plans for project and implementation of this study. None of them are asked to advise on interpretation of results. The results will be disseminated to the general population through public presentations by the authors.

\section{DISCUSSION}

In the systematic reviews and meta-analyses that focused on clinical outcomes after using aliskiren alone, some of the results are widely divergent. To the best of our knowledge, this is the first study that systematically summarises the effectiveness, safety and tolerability of aliskiren monotherapy clinically. When sufficient data are available, we will stratify our comparisons by dosage or specific categories of the medications. We will also calculate $95 \%$ PI in order to provide more unbiased and convincing results.

The limitations of our study are the heterogeneity and quality of the selected reviews. To address the limitations, we will reanalyse each outcome using the random effects model. We will also use AMSTAR 2 to evaluate quality of studies that we will include. Furthermore, these two factors will be carefully considered when interpreting the results.

\section{Ethics and dissemination}

Ethical approval is not required for the study, as we only collected data from available materials. This umbrella review will be also submitted to a peer-reviewed journal for publication.

Contributors JS carried on the conception and construction of this protocol. JL developed the search strategy. QJ and YW compared and found the best tools for assessing possible bias and evaluating quality of included reviews. QZ wrote the protocol. JS reviewed and amended the draft of the protocol. FL added grammar editing and conceptual clarification. All authors read and approved of the final manuscript.

Funding This work was supported by the Department of Education of Zhejiang Province, China [Grant numbers Y201635273].

Competing interests None declared.

Patient consent for publication Not required.

Provenance and peer review Not commissioned; externally peer reviewed.

Open access This is an open access article distributed in accordance with the Creative Commons Attribution Non Commercial (CC BY-NC 4.0) license, which permits others to distribute, remix, adapt, build upon this work non-commercially, and license their derivative works on different terms, provided the original work is properly cited, appropriate credit is given, any changes made indicated, and the use is non-commercial. See: http://creativecommons.org/licenses/by-nc/4.0/.

ORCID iD

Qiyuan Zhao http://orcid.org/0000-0003-2854-0845

\section{REFERENCES}

1 Whelton PK, Carey RM, Aronow WS, et al. 2017 ACC/AHA/AAPA ABC/ACPM/AGS/APhA/ASH/ASPC/NMA/PCNA guideline for the prevention, detection, evaluation, and management of high blood pressure in adults: a report of the American College of Cardiology/ American heart association Task force on clinical practice guidelines. Hypertension 2018;71:e13-115.

2 Muntner P, Carey RM, Gidding S, et al. Potential U.S. Population Impact of the 2017 ACC/AHA High Blood Pressure Guideline. J Am Coll Cardiol 2018;71:109-18.

3 Egan BM, Li J, Hutchison FN, et al. Hypertension in the United States, 1999 to 2012: progress toward healthy people 2020 goals. Circulation 2014;130:1692-9.

4 Yoon SS, Fryar CD, Carroll MD. Hypertension prevalence and control among adults: United States, 2011-2014. NCHS Data Brief 2015;220.

5 Vos T, Flaxman AD, Naghavi M, et al. Years lived with disability (YLDs) for 1160 sequelae of 289 diseases and injuries 1990-2010: a systematic analysis for the global burden of disease study 2010 . The Lancet 2012;380:2163-96.

6 Heidenreich PA, Albert NM, Allen LA, et al. Forecasting the impact of heart failure in the United States: a policy statement from the American heart association. Circ Heart Fail 2013;6:606-19.

7 Jorsal A, Wiggers H, McMurray JJV. Heart failure: epidemiology, pathophysiology, and management of heart failure in diabetes mellitus. Endocrinol Metab Clin North Am 2018;47:117-35.

8 Sureshkumar KK. Renin inhibition with aliskiren in hypertension: focus on aliskiren/hydrochlorothiazide combination therapy. Vasc Health Risk Manag 2008;4:1205-20.

9 Flather MD, Yusuf S, Køber L, et al. Long-Term ACE-inhibitor therapy in patients with heart failure or left-ventricular dysfunction: a systematic overview of data from individual patients. The Lancet 2000;355:1575-81.

10 Musini VM, Lawrence KA, Fortin PM, et al. Blood pressure lowering efficacy of renin inhibitors for primary hypertension. Cochrane Database Syst Rev 2017;4:CD007066.

11 Rao MS. Inhibition of the renin angiotensin aldosterone system: focus on aliskiren. JAPI 2010;58:102-8.

12 Verdecchia P, Angeli F, Mazzotta G, et al. Angiotensin converting enzyme inhibitors and angiotensin receptor blockers in the treatment of hypertension: should they be used together? Curr Vasc Pharmacol 2010;8:742-6.

13 Andersen $\mathrm{K}$, Weinberger $\mathrm{MH}$, Egan B, et al. Comparative efficacy of aliskiren monotherapy and ramipril monotherapy in patients with stage 2 systolic hypertension: subgroup analysis of a double-blind, active comparator trial. Cardiovasc Ther 2010;28:344-9.

14 Parving $\mathrm{H}-\mathrm{H}$, Persson F, Lewis JB, et al. Aliskiren combined with losartan in type 2 diabetes and nephropathy. N Engl J Med 2008;358:2433-46.

15 Pool JL, Schmieder RE, Azizi M, et al. Aliskiren, an orally effective renin inhibitor, provides antihypertensive efficacy alone and in combination with valsartan. Am J Hypertens 2007;20:11-20.

16 Bangalore S, Kumar S, Messerli FH. When conventional heart failure therapy is not enough: angiotensin receptor blocker, direct renin inhibitor, or aldosterone antagonist? Congest Heart Fail 2013;19:107-15.

17 Catalá-López F, Macías Saint-Gerons D, González-Bermejo D, et al. Cardiovascular and renal outcomes of renin-angiotensin system blockade in adult patients with diabetes mellitus: a systematic review with network meta-analyses. PLoS Med 2016;13:e1001971.

18 Chen $Y$, Meng L, Shao H, et al. Aliskiren vs. other antihypertensive drugs in the treatment of hypertension: a meta-analysis. Hypertens Res 2013;36:252-61.

19 Zhang J-T, Chen K-P, Guan T, et al. Effect of aliskiren on cardiovascular outcomes in patients with prehypertension: a meta-analysis of randomized controlled trials. Drug Des Devel Ther 2015;9:1963-71.

20 Zheng SL, Roddick A, Ayis S. P2093Effects of aliskiren on mortality, cardiovascular outcomes and adverse events in diabetics with cardiovascular disease or risk: a systematic review and metaanalysis of 13,395 patients. Eur Heart $J$ 2017;38:400-6.

21 Verdecchia P, Angeli F, Mazzotta G, et al. Aliskiren versus ramipril in hypertension. Ther Adv Cardiovasc Dis 2010;4:193-200. 
22 Poole R, Kennedy OJ, Roderick P, et al. Coffee consumption and health: umbrella review of meta-analyses of multiple health outcomes. BMJ 2017;359:j5024-18.
23 Shea BJ, Reeves BC, Wells G, et al. AMSTAR 2: a critical appraisal tool for systematic reviews that include randomised or nonrandomised studies of healthcare interventions, or both. BMJ 2017;358:j4008-8. 